This value is far smaller than could reasonably be expected, as the reaction is slightly exothermic and should take place with considerable ease with fast neutrons. This result must mean either that one reaction is for some reason extraordinarily improbable, or that the activity observed by Pollard and McMillan is in fact not $\mathrm{Be}^{10}$, since the decay constant determined by Pollard can scarcely be in error by several powers of 10. Support for this last suggestion lies in the circumstance that the activity from a beryllium target has not been identified chemically by the previous observers.

I am greatly obliged to Dr. N. Feather for the loan of his counter and to Mr. W. Birtwhistle for technical assistance.

Note added on proof : R. D. O. Neal and M. Goldhaber (Phys. Rev., 57, 1086) have shown that the observed activity of $\mathrm{Be}$ on deuteron bombardment is due to $\mathrm{H}^{3}$.

E. BRETSCHER.

Cavendish High Tension Laboratory, Cambridge.

June 29.

${ }^{1}$ Phys. Rev., 49, 875 (1936).

${ }^{2}$ Phys. Rev. 57, 241 (1940).

${ }^{3}$ See summary Jordan, P., Ergeb. exakt. Nalurw., 16, 90.

\section{Cosmic Ray Intensities in Relation to Cyclones and Anticyclones}

Is a previous letter ${ }^{1}$, we showed that cosmic ray intensities undergo characteristic changes during the passage of cyclones. The computations were carried out for cyclones irrespective of any seasons. Since, however, the paths of cyclones are generally subjected to seasonal variations, it is possible that the seasonal effect of cosmic ray intensities is superimposed on the results there obtained. We therefore followed the procedure as mentioned below, which enables one to eliminate the seasonal effect. The results thus obtained corroborate the previous conclusions. The same method was also applied to anticyclones.

We used nearly the same hourly means of cosmic ray intensities, $\mathbf{3 6 2}$ in number, observed during the traversal of the same 11 typical cyclones as were chosen in the previous letter, the only minor difference being the rejection of two unimportant cases which were disturbed by magnetic storms. The same method of representation on a polar diagram was

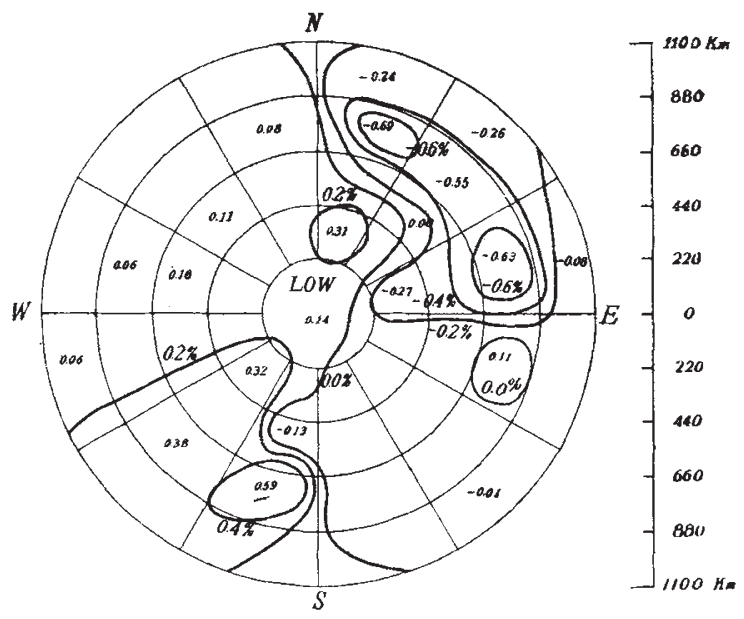

Fig. 1.

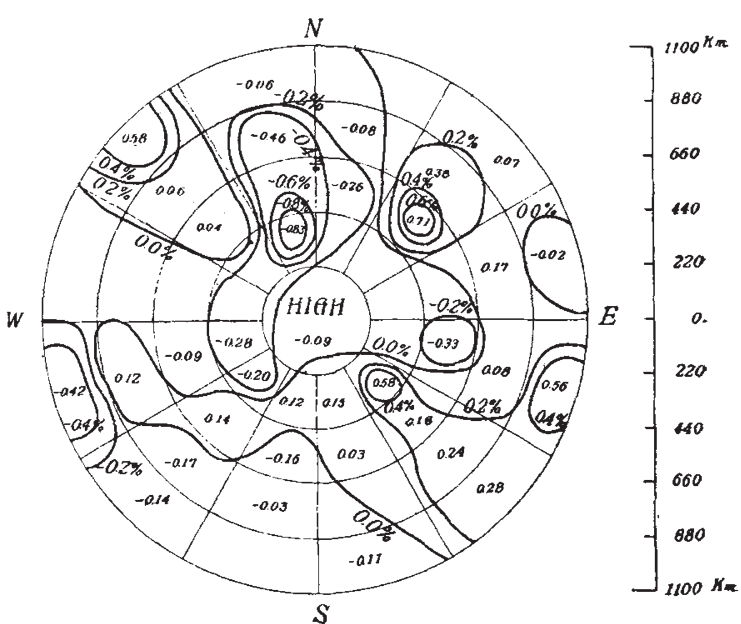

Fig. 2.

also followed as before. Instead of writing down, however, the values of cosmic ray intensities themselves on the sections of the diagram corresponding to the positions of Tokyo, we put down their percentage deviations from the mean intensities during the passage of individual cyclones. In this way we obtained the mean percentage deviation for each section as given in Fig. 1, which shows the variation of cosmic ray intensities due to cyclonic migration free from the seasonal effect. As can be seen from the figure, the general features are nearly the same as in Fig. 2 of the previous note, although the magnitude of the variation is less, namely, \pm 0.5 per cent in this case.

Exactly the same procedure was adopted for $\mathbf{2 5}$ cases of anticyclones, the centres of which were determinable within a radius of about $1,100 \mathrm{~km}$. from Tokyo as before. Corresponding hourly means of cosmic ray intensities, 598 in number, were treated in the same way as above. The final results thus obtained are given in Fig. 2, in which the distribution. of intensity deviations is more complicated than that of Fig. I. Since the anticyclone traverses the area in question from west-north-west to east-south-east, carrying cold air masses of polar origin in the front and warm air masses of tropical origin in the rear, the general feature of Fig. 2 seems again to illustrate the fact that the arrival of $a$ cold air mass tends to increase the intensity of the hard component of cosmic rays, while that of a warm air mass tends to decrease it, in conformity with the instability of the meson. The high values, however, in the north-west quadrant as well as in the south-west quadrant, as: shown in Fig. 2, seem to show that the warm rear of the anticyclone is again followed by a cold air mass. Whether this is due to the cold front of the succeeding anticyclone or to the specific character of the anticyclone itself will be seen in the future.

This work was done as a part of the programme of the Cosmic-Ray Sub-Committee of the Japan Society for the Promotion of Scientific Research.

$$
\begin{aligned}
& \text { Y. Nishina. } \\
& \text { Y. Sekido. } \\
& \text { H. Simamura. }
\end{aligned}
$$

Institute of Physical and Chemical Research, Tokyo.

\section{H. Arakawa.}

Central Meteorological Observatory, Tokyo. April 22.

${ }^{1}$ Nishina, Y., Arakawa, H., Sekido, Y., and Simamura, H., NATURE, 145, $703(1940)$. 